\title{
DOI 10.18699/BGRS/SB-2020-158 \\ Polygenic transmission disequilibrium of slightly-deleterious variants in Down syndrome trios
}

\author{
Kseniia Sholokhova \\ Center for Mitochondrial Functional \\ Genomics \\ IKBFU, Kaliningrad, Russia \\ Viktor Shamansky \\ Center for Mitochondrial Functional \\ Genomics \\ IKBFU, Kaliningrad, Russia
}

\begin{abstract}
A trisomy 21 (T21) is associated with high, up to $80 \%$ miscarriage rates. Yet the genetic component of this selection is unknown - why some fetuses with $\mathrm{T} 21$ can be liveborn, while others are miscarried. Here we hypothesize that genome-wide burden of slightly-deleterious variants in the T21 genome can affect the outcome. To test this hypothesis we analyze genomic data from families with live-born offspring with T21. In order to uncover patterns of embryonic selection against fetuses with $\mathrm{T} 21$ we need to analyze which set of alleles is over- or under- inherited from parents to offspring with T21. For this, we calculate individual predisposition of each member of a trio to different phenotypes (mainly different diseases). These genetic risks, or polygenic risk scores (PRS) are calculated by summing up risk alleles, that are weighted by effect sizes derived from GWAS analyzes. Comparing the PRS of parents with the PRS of offspring we can uncover disequilibrium in the transmission of certain risks. Understanding of molecular mechanisms of over- and undertransmission of alleles is of great importance because they can determine the viability of $\mathbf{T} 21$ fetuses. Discovered in this way non-randomly transmitted classes of polymorphisms can help us to point out molecular signatures of phenotypes associated with T21 as well as may potentially help to find new ways of treatment.
\end{abstract}

Keywords - PRS, T21, Down syndrome, polymorphisms, slightly-deleterious variants, transmission disequilibrium

Introduction

To date, trisomy 21 is the most common chromosomal abnormality occurring in humans. It is caused by a meiotic nondisjunction event with subsequent presence of all or part of the third copy of chromosome 21. Moreover, trisomy 21 is associated with high, up to $80 \%$ miscarriage rates [1].

Down syndrome phenotypes are induced by trisomy 21 but they can be modified by numerous slightly deleterious variants located on both chromosome 21 [2] and all other chromosomes [3]. Here using PRS approach we try to find a set of alleles which can modify the effect of T21. Overtransmitted alleles we interpret as protective ones, which can partially compensate the effect of T21, while undertransmitted alleles we interpret as deleterious ones, which aggravate the effect of $\mathrm{T} 21$.

\section{Methods}

Our main dataset is 628 genotyped trios (two parents and live-born DS offspring in each trio). Using all DNA polymorphisms, associated with 50 different traits (largescale studies, $\mathrm{N}>50,000)$ such as body mass index (BMI), coronary artery disease, blood pressure, type 2 diabetes,

\author{
Konstantin Popadin \\ School of Life Sciences \\ Ecole Polytechnique Federale de \\ Lausanne, Lausanne, Switzerland \\ Center for Mitochondrial Functional \\ Genomics, IKBFU, Kaliningrad, Russia \\ konstantinpopadin@gmail.com
}

etc.), we calculated a polygenic risk score (PRS) for each member of each trio. Below we describe step by step how we calculated PRS and estimated transmission of these risks from parents to T21 offspring.

\section{(i) PRS}

For finding complex diseases which affect DS we first counted polygenic risks scores. A polygenic risk score (PRS) is a sum of trait-associated alleles weighted by effect sizes estimated from a genome-wide association study.

We counted PRS in 4 different ways:

(1) without in-house strict clumping \& thresholding the number of SNPs, on a full sample, for all trios - in this case, the soft clumping \& thresholding built-in PRSice2.2.8 was used;

(2) by in-house strict clumping \& thresholding the number of SNP, calculation of PRS on a complete sample, for all trios by simple summation of the products beta $* \mathrm{~N}$, where $\mathrm{N}$ is the number of alleles of the alternative genotype;

(3) by in-house strict clumping \& thresholding, calculating PRS for each triple using PRSice2.2.8 (clumping \& thresholding built into PRSice2.2.8 was disabled by the -no-clump option);

(4) by in-house strict clumping \& thresholding, calculating PRS on a full sample, for all triples using PRSice2.2.8 (the built-in clipping \& thresholding in PRSice2.2.8 was disabled by the -no-clump option).

The highest qualitative correspondence, according to the variation of CliffDelta and CohenD, with the hypothesis that the median PRS of parents more than in children with Down syndrome was obtained only by using the simplest method (2).

It has to be said that we measured the effect size of the differences between the PRS distributions of parents and children with Down syndrome using Cliff's Delta and Cohen D - all 4 methods yielded negligible differences between the compared PRS distributions.

In addition, it must be said that we tried to calculate the statistical significance of differences in the distributions of PRS of parents and PRS of children with Down syndrome using Welch t-test and U-test. Statistically significant (however marginally significant) differences in the distributions were obtained exclusively by analysis by method (1), however, the variations of Cliff's Delta and Cohen D, not to mention PRS of children with Down syndrome or PRS of parents, in this case were inconsistent with each other. 


\section{(ii) Transmission disequilibrium}

The transmission of burden of slightly deleterious variants can be estimated by polygenic transmission disequilibrium test (pTDT) [4]. This approach allows to point out how various genetic variants can be over- and under-transmitted from parents to offspring.
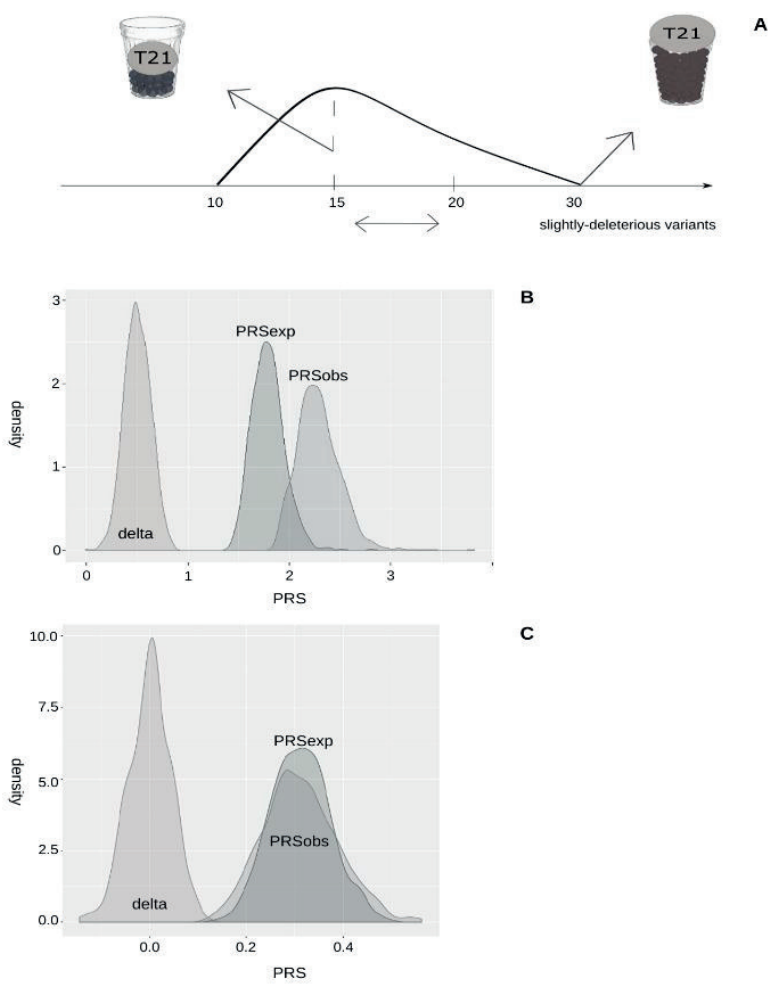

Fig. 1. The concept of inheritance of slightly deleterious variants.

A-a number of slightly-deleterious variants determines whether the fetus with T21 will be live-born (left cup with some space) or a miscarriage (overflowing right cup);

B- fictional model with transmission disequilibrium, where delta is different from 0, PRSexp - expected RPS (based on parents' genotypes), PRSobs - observed PRS (based on an offspring's genotype);

C- results as a PRS for schizophrenia, schizotypal and delusional disorders, where delta equals 0, PRSexp - expected RPS (based on parents' genotypes), PRSobs - observed PRS (based on an offspring's genotype).
When PRSs for both parents and offspring are counted we compare average (mid-parent score) and/or whole distributions with the PRS of offspring. The contrast between the scores will shed the light whether genetic predisposition to a given phenotype was randomly transmitted to offspring (i. e. contrast is close to zero) or over- / under- transmitted.

\section{Results}

Currently, analyses of transmission of all calculated risks demonstrated the expected neutral inheritance from parents to the offspring (average delta $=0$ ), as in fig.1C. It is in line with the absence of strong genetic selection for or against the T21 during embryogenesis. This can be explained either by real absence of genetic selection (meaning that live-born or miscarried T21 can be purely random or depends on environment) or by the fact that we didn't find yet T21 - relevant phenotype. Thus we continue to look for polygenic transmission disequilibrium in other phenotypes.

In addition to this, we plan to annotate specific targets of DS embryonic selection in order to point out the strongest genetic variants, genes and gene networks which affect survival probability of individuals with Down Syndrome.

\section{Acknowledgment}

This work has been supported by the 5 Top 100 Russian Academic Excellence Project at the Immanuel Kant Baltic Federal University, by the Russian Foundation for Basic Research grant 18-29-13055.

\section{References}

[1] Nussbaum RL, McInnes RR, Willard HF. 2004. Thompson \& Thompson genetics in medicine, revised reprint, 6th ed. Saunders, Philadelphia, PA

[2] Sailani MR, Makrythanasis P, Valsesia A, Santoni FA, Deutsch S, Popadin K, Borel C, Migliavacca E, Sharp AJ, Duriaux Sail $\mathrm{G}$, et al. 2013. The complex SNP and CNV genetic architecture of the increased risk of congenital heart defects in Down syndrome. Genome Res 23: 1410-1421.

[3] Letourneau A, Santoni FA, Bonilla X, Sailani MR, Gonzalez D, Kind J,Chevalier C, Thurman R, Sandstrom RS, Hibaoui Y, et al. 2014. Domains of genome-wide gene expression dysregulation in Down's syndrome. Nature 508: 345-350.

[4] Weiner et al 2017 Polygenic transmission disequilibrium confirms that common and rare variation act additively to create risk for autism spectrum disorders. Nature Genetics volume 49, pages $978-998$ 